Journal of Applied Pharmaceutical Science Vol. 6 (07), pp. 125-132, July, 2016

Available online at http://www.japsonline.com

DOI: $10.7324 /$ JAPS.2016.60719

ISSN 2231-3354 (cc) EY-NC-SA

\title{
Self microemulsefying and non-self microemulsefying liquisolid tablet of felodipine
}

\author{
Gehad S. Khorshed*, Gamal M. El-Maghraby \\ Department of Pharmaceutical Technology, College of Pharmacy, University of Tanta, Tanta, Egypt.
}

\begin{tabular}{|c|c|}
\hline ARTICLE INFO & ABSTRACT \\
\hline Article history: & \multirow{10}{*}{$\begin{array}{l}\text { Liquisolid formulation involves dissolution or dispersion of the drug in a liquid before formulation of solid } \\
\text { dosage form using solid adsorbent as a carrier. The liquid component usually utilizes simple solvent system. Self } \\
\text { emulsifying liquids can provide another alternative liquid for enhanced dissolution. The objective of this work } \\
\text { was to develop and evaluate non-self emulsifying and self emulsifying liquisolid tablet for enhanced dissolution } \\
\text { rate of felodipine. The former utilized polyethylene glycol } 400 \text { which was the best solvent and the later } \\
\text { employed a mixture of castor oil with labrasol and transcutol as self emulsifying system. A mixture of avicel } \\
\text { PH } 102 \text { and aerosil } 200 \text { was used as solid adsorbent with croscarmellose being used as superdisintegrent. } \\
\text { Formulation of the drug as liquisolid tablets enhanced the dissolution rate compared with the standard tablet } \\
\text { prepared using the unprocessed drug powder with the same tablet excipients. The self emulsifying tablet showed } \\
\text { faster release pattern compared with the non-self emulsifying tablet. The thermal analysis studies indicated the } \\
\text { presence of the drug in a solution form in the tablet formulations. In conclusion liquisolid tablet formulation can } \\
\text { enhance the dissolution rate of felodipine with self emulsifying liquid system being more efficient. }\end{array}$} \\
\hline Received on: $28 / 08 / 2015$ & \\
\hline Revised on: 27/10/2015 & \\
\hline Accepted on: & \\
\hline Available online: $28 / 07 / 2016$ & \\
\hline Key words: & \\
\hline Felodipine dissolution; & \\
\hline SEDDS; thermal analysis; & \\
\hline superdisintegrant. & \\
\hline & \\
\hline
\end{tabular}

\section{INTRODUCTION}

Felodipine is a dihydropyridine calcium channel blocker which is used for treatment of hypertension, angina pectoris and congestive heart failure (Saltiel et al., 1988). Felodipine is a class 2 drug according to the Biopharmaceutical Classification System (BCS), meaning that it is poorly soluble and highly permeable. The major problem of this drug is its poor oral bioavailability with only $15 \%$ of the dose reaching the systemic circulation after oral administration. This was mainly attributed to extensive first pass metabolism with the poor dissolution adding to the problem (Hariprassana et al., 2010). These features suggest that drug dissolution can be the rate limiting step for drug absorption (Kuchekar and Narkhede, 2007). Accordingly, enhancing the dissolution rate of felodipine can be a promising strategy for enhancing its oral bioavailability.

* Corresponding Author

Gehad S Khorshed, Department of Pharmaceutical Technology,

College of Pharmacy, University of Tanta, Tanta. Egypt

Email:gehad_khorshed@yahoo.com
The benefit will become even greater if the developed system and/or the selected excipient can reduce the presystemic metabolism. Alternative strategies have been employed to enhance dissolution rate of drugs. These include micronization, formation of solvates, complexation and preparation of solid dispersions with hydrophilic polymers (Kim and Park, 2004). Liquisolid system is another promising technique that is expected to increase the dissolution rate and hence the oral bioavailability of drugs (Spireas et al., 1998). A liquisolid system is formed by dissolving or suspending the drug in a suitable liquid resulting in the formation of a "liquid medication". This is then converted into a dry looking, non adherent, free flowing and readily compressible powder. This is achieved by blending the liquid medication with suitable carrier and coating materials (Javadzadeh et al., 2005). Many grades of cellulose, starch, lactose, etc. can be used as carriers with silicas of very fine particle size can be used as coating materials (Tayel et al., 2008). The presence of coating materials is essential to provide flowable powder mixtures (Nokhodchi et al., 2010). It was found that the smaller the drug concentration in the liquid medication, the more rapid the release rates. 
This was explained on the bases that high drug concentration can lead to precipitation within the silica pores (Khaled, 1998). Silicas possessing large surface areas, and microcrystalline cellulose of small particle size and granular grades resulted in a good flow and compression properties, producing acceptable tablets (Spireas et al., 1992). The advantages of this technique include Simplicity, low cost and suitability of scaling up to industrial production (Spireas and Bolton, 1998). The liquisolid technique has been successfully employed to improve the in-vitro release of poorly water soluble drugs as hydrocortisone (Spireas et al., 1998), methylclothiazide (Spireas et al., 1999), hydrochlorothiazide (Khaled, 1988), prednisolone (Spireas and Sadu, 1998). The advantages of this technique was extended by manipulation of the nature of the liquid system so as to develop a system for enhanced dissolution, enhanced permeability and/or reduced presystemic metabolism. Recently, much attention has been given to self-emulsifying drug delivery system (SEDDS) which is believed to increase the dissolution rate, and absorption of drugs with a potential for reduction of the presystemic metabolism (Charman et al., 1992). SEDDS are isotropic mixtures of oil, surfactant and usually cosurfactant or cosolvent. These systems form stable microemulsion or coarse emulsion upon dispersion in aqueous media (Hauss, 2007). Accordingly, the objective of this work was to develop and evaluate selfemulsifying and non self-emulsifying liquisolid tablet felodipine.

\section{MATERIALS AND METHODS}

\section{Materials}

Felodipine was purchased from Liangshan Bangda Pharmaceutical Co, Ltd, China. Avicel PH 102, Aerosil 200 and croscarmellose were kindely provided by Sigma Pharmaceutical Industries, Quesna, Egypt. Polyethylene glycol 400 was purchased from $\mathrm{BDH}$, Poole, England. Labrasol and Transcutol were obtained as a gift from Gattefosse, France. Methyl alcohol, Tween 80, propylene glycol, olive oil and oleic acid were purchased from El Nasr Pharmaceutical Chemical Co., Cairo, Egypt. Castor oil was purchased from Iso-chem., Egypt.

\section{Construction of the calibration curve}

Drug analysis employed UV spectrophotometry with drug concentration being determined at 238nm (Cardoza and Amin, 2002). This utilized a Thermo Fisher Scientific spectrophotometer, Madison, WI, USA. Serial concentrations of the drug were prepared in methanol. These were used to construct the calibration curve of the drug which was linear over the concentration range of $2-24 \mu \mathrm{g} / \mathrm{ml}\left(\mathrm{R}^{2}=0.997\right)$.

\section{Solubility screening}

The solubility of the drug in the liquid part of liquisolid system is essential parameter for selection of the solvent system. The same applies for selection of the oil in case of SEDDS. Accordingly, the solubility of felodipine was determined in a series of liquids. Excess amount of drug was added to each of the tested liquids. The resultant suspension was left to equilibrate at ambient temperature under magnetic stirring for 72 hours, at the end of which excess drug was separated by centrifugation. The solubility was then determined spectrophotometrically after suitable dilution with methanol. A blank was prepared by similar dilution of the corresponding drug free liquid system.

\section{Determination of phase behavior}

Castor oil was selected as the oil phase as it solubilized the largest amount of the drug. labrasol and transcutol were used as the surfactant/cosurfactant system and were employed at a ratio of 1:1 w/w (Singh et al., 2009). Mixtures of oil with surfactant or surfactant-cosurfactant system was prepared at ratios of $1: 19,2: 18$, 2.5:17.5, 3:17, 3.5:16.5, 4:16, 4.5:15.5, 5:15, 5.5:14.5, 6:14, 8:12 and 10:10; oil to surfactant system respectively. These mixtures were vortex mixed before mixing $50 \mathrm{mg}$ with $20 \mathrm{ml} 0.1 \mathrm{~N} \mathrm{HCl}$. The resulting dispersion was mixed and left to equilibrate before visual inspection and analysis for transmittance at $600 \mathrm{~nm}$ on UV/Visible spectrophotometer.

\section{Preparation of liquisolid tablet \\ Determination of the angle of slide of Aerosil 200 and Avicel PH 102}

Constant weight of each powder (10 g) was placed at one end of a glass plate. This end was gradually elevated to create an angle with the horizontal plane. The process continued to reach an angle at which the powder was about to slide. This angle was measured and was recorded as the angle of slide. The recorded angle of slide was taken as a measure for the flow properties of the powders. An angle of slide corresponding to $33^{\circ}$ was considered as the maximum angle indicating acceptable flow for liquisolid tablet manufacture (Tayel et al., 2008).

\section{Determination of flowable liquid retention potential for Aerosil 200 and Avicel PH 102 (Ф -value)}

Increasing amounts of the liquid system were mixed well with $10 \mathrm{~g}$ of either Aerosil 200 or Avicel PH 102 powder. The angle of slide was determined for each of these mixtures. The $\boldsymbol{\Phi}$ value of each mixture was calculated using the following equation: $\boldsymbol{\Phi}$-value = weight of liquid/weight of solid

The $\boldsymbol{\Phi}$-values were plotted versus the corresponding angles of slide. The $\boldsymbol{\Phi}$-values corresponding to an angle of slide of $33^{\circ}$ was taken as the flowable liquid retention potential (Tayel et al., 2008).

\section{Preparation and mixing of the powders}

The amounts of excipients depended on their $\boldsymbol{\Phi}$-values, as well as liquid load factors $\left(\mathrm{L}_{\mathrm{f}}\right)$. Having Aerosil 200 as the coating material and Avicel PH 102 as the carrier at a ratio of 1:10, the $\mathrm{L}_{\mathrm{f}}$ was calculated using the following equation.

$\mathrm{L}_{\mathrm{f}}=\boldsymbol{\Phi}$ of the carrier+ $\boldsymbol{\Phi}$ of the coat (1/R) (Tayel et al., 2008). Where, $\mathrm{R}$ is the weight ratio of carrier $(\mathrm{Q})$ to the coating material (q) present in the formulation. 
The amounts of excipients used to prepare the tablets are related to the amount of liquid medication (W) through the 'Liquid Load Factor' $\left(\mathrm{L}_{\mathrm{f}}\right)$ as shown in the following equation:

$$
\mathrm{L}_{\mathrm{f}}=\mathrm{W} / \mathrm{Q} \text {. }
$$

The selected weight of medicated liquid (SEDDS or polyethylene glycol 400) was mixed with the carrier followed by the coating material to produce free flowing powder. This order of mixing is believed to produce the most optimal release rate. Croscarmellose was used as superdisintigrant and was included at a concentration of $5 \% \mathrm{w} / \mathrm{w}$ in the tablet. The final mixture was compressed using single punch tableting machine. For the self emulsifying liquisolid tablet $14 \mathrm{~mm}$ rounded punch with flat surface was used while $12 \mathrm{~mm}$ rounded punch with flat surface was used for the non self emulsifying liquisolid tablets and the control tablets. (Royal Artist, Kapadia Industrial Estate, BLDG, Mumbai, India).

\section{Determination of dissolution rate}

The USP paddle method was used for dissolution studies. This employed apparatus 2 (Copley Sceintific, Model: NE4-COP, United Kingdom). The dissolution medium was $500 \mathrm{ml}$ of sodium phosphate buffer ( $\mathrm{pH}$ 6.5) with $1 \% \mathrm{w} / \mathrm{v}$ sodium lauryl sulphate being included to maintain sink conditions. This was maintained at $37 \pm 1{ }^{\circ} \mathrm{C}$ with the rate of stirring being $50 \mathrm{rpm}$ (USP, 2009). The drug $(10 \mathrm{mg})$ was added in the form of untreated powder, dispersion in SEDDS or liquisolid tablets. Samples $(5 \mathrm{ml})$ were taken at appropriate time intervals $(5,10,15,30,45,60,90,120$ min). These were immediately filtered through a $0.45 \mu \mathrm{m}$ Millipore filter. Fresh dissolution medium was added to compensate for the samples to maintain a constant volume. The drug concentration in each sample was determined spectrophotometrically at $238 \mathrm{~nm}$. The study was conducted in triplicates. The cumulative amount dissolved expressed as percentage of the total drug added was plotted as a function of time to produce the dissolution profile. The dissolution efficiency was calculated from the area under the curve of the dissolution profile which was calculated using nonlinear trapezoidal method and was demonstarted as a percentage of the area of the rectangle corresponding to $100 \%$ dissolution in the same time (El Maghraby and Elsergany, 2014).

\section{Evaluation of liquisolid tablets \\ Uniformity of weight}

The USP weight variation test was conducted by weighing 20 tablets individually, calculating the average weight and comparing the individual tablet weights to the average. The allowed percentage deviation is $5 \%$. The tablets meet the USP test if no more than two tablets are outside the limit and no tablet deviates by more than twice the limit according to USP.

\section{Tablet friability}

The friability of the tablets was measured in a Friabilator (Erweka Apparatebau-G.m.b.H Model: TA3, Western Germany). A pre-weighed tablet sample (30 tablets) was placed in the friabilator and subjected to 100 revolutions. The tablets were carefully dedusted and weighed again. The friability was calculated as the percentage loss which should not exceed $1 \%$ according to USP.

\section{Drug content uniformity}

To ensure uniform potency, a content uniformity test was applied by random selection of 30 tablets. At least 10 tablets of them were individually subjected to drug content determination. Each tablet was powdered and dispersed in methanol with aid of sonication to dissolve the drug. This was filtered before determining the drug content by UV spectrophotometer after suitable dilution. The tablets were considered acceptable if the content of each of the 10 tablets was in the range of $85-115 \%$ of the labeled amount of felodipine. The batch fails if more than one tablet is outside the limit or if any tablet contain $<75 \%$ or $>125 \%$ of the labeled content. If only one tablet was outside the limit, the remaining 20 tablets must be analyzed individually and all of them should be within the limit according to USP.

\section{Disintegration test}

The test was carried out on six tablets using tablet disintegration tester (Copley Scientific, Model: NE4-COP, United Kingdom) using $0.1 \mathrm{~N} \mathrm{HCl} \mathrm{pH} 1.2$ as a disintegration media and the time taken for complete disintegration of the tablet was recorded.

\section{Hardness test}

The hardness of 20 tablets was measured using hardness test machine (Copley Scientific, Model: TBF 1000, United Kingdom).

\section{Differential Thermal Analysis test}

Thermograms of the pure drug, avicel, aerosil, and the liquisolid formulations were recorded using a differential thermal analyzer (DTA) (Perkin Elmer, STA 6000, France). Samples equivalent to 2-3 $\mathrm{mg}$ of the drug were loaded into aluminum pans and the lids were crimped. The thermal behavior of each sample was investigated under nitrogen at a heating rate of $10{ }^{\circ} \mathrm{C} /$ minutes, covering temperature ranges of $30-300{ }^{\circ} \mathrm{C}$. Data analysis was conducted using TA-60WS thermal analysis software.

\section{RESULT AND DISCUSSION}

\section{Solubility screening}

Liquisoild systems involve solubilizing the drug in a suitable pharmaceutical solvent before incorporation in a solid dosage form. This requires screening of drug solubility in different solvents (Javadzadeh et al., 2008). Considering the aim of this work which was to develop and evaluate self emulsifying and nonself emulsifying liquisolid tablets for enhanced dissolution of felodipine, it was necessary to perform solubility screening in various solvents, oils, surfactants and cosurfactants. The results of this solubility studies were used for selection of the liquid 
medication for the non-self emulsifying liquisoild tablets as well as the components of the self emulsifying system. Table 1 presents the equilibrium solubility of felodipine in various solvents, oils, surfactants and cosurfactants. With respect to the solubility of drug in different solvents, polyethylene glycol 400 solubilized $233 \mathrm{mg}$ of the drug in each milliliter of the solvent. This data is similar to that recorded by (Basaliou et al., 2013). With respect to the solubility in the surfactant cosurfactant systems, Labrasol solubilized the highest amount of drug compared to other surfactants with transcutol being the most effective with respect to solubility, compared to other cosurfactant. Transcutol was similarly shown to be efficient solvent for felodipine (Ansari et al., 2014). For the oily phase two vegetable oils were evaluated for the solubilizing capacity to felodipine. Castor oil was found to a better solvent for the drug compared to olive oil. Based on the results of this solubility and the miscibility of the surfactant cosurfactant system with the castor oil, the components of SEDDS were castor oil with labrasol and transcutol. Similar components were successfully used to prepare SEDDS containing castor oil (Singh et al., 2009).

Table 1: Equilibrium solubility of felodipine in various solvents, oils surfactants and cosurfactants

\begin{tabular}{ll}
\hline Liquids & Solubility $(\mathbf{m g} / \mathbf{m l})$ \\
\hline Polyethylene glycol 400 & $233.47 \pm 2.33$ \\
Propylene glycol & $37.98 \pm 3.37$ \\
Oleic acid & $18.53 \pm 3.1$ \\
Tween 80 & $137.74 \pm 3.3$ \\
Labrasol & $163.795 \pm 1.17$ \\
Transcutol & $361.2 \pm 2.18$ \\
Castor oil & $47.08+0.61$ \\
Olive oil & $19.14 \pm 0.67$ \\
SEDDS* & $254.27 \pm 0.97$ \\
\hline
\end{tabular}

*SEDDS is a selfemulsifying system comprising castor oil, labrasol and transcutol $(1: 2: 2)$

\section{Determination of the phase behavior}

SEDDS should form emulsions or microemulsions under the gentle agitation of the digestive motility in the stomach and intestines. However, for this process to take place the ratio of oil to the surfactant cosurfactant system should be optimum (Gangineni et al., 2013). Accordingly it was necessary to determine the phase behavior of the oil and surfactant/cosurfactant systems when gently mixed with the gastric fluid. To achieve this, Labrasol and transcutol were mixed at 1:1 weight ratio. This mixture was mixed with castor oil at various ratios. The resulting systems were tested for their ability to form emulsion or microemulsion. The mixtures were mixed with aqueous phase and the resulting system was characterized visually and spectrophotometry. Table 2 presents the recorded absorbance values after introduction of each mixture to the aqueous phase. All the tested mixtures were able to form SEDDS visually but the turbidimetric studies revealed variation in the absorbance. More turbid system was taken as indication of larger globule size. This was taken into consideration in selection of the SEDDS for preparation of liquisolid tablets with the systems producing less turbid emulsion being considered. Thus the selected ratio for the microemulsion of castor oil, labrasol and transcutol was 1:2:2, respectively. This system was shown to be good solvent for felodipine with solubility of the drug being $254.27 \mathrm{mg} / \mathrm{ml}$ in such system. This system was used to develop the liquisolid tablet according to the formulation presented in Table 3 .

Table 2: Phase behavior of castor oil-labrasol-transcutol systems after dilution with $0.1 \mathrm{~N}$ hydrochloric acid.

\begin{tabular}{ccccc}
\hline Castor oil & Labrasol & Transcutol & $\begin{array}{c}\text { Visual } \\
\text { Appearance }\end{array}$ & $\begin{array}{c}\text { Recorded } \\
\text { absorbance }\end{array}$ \\
\hline 1 & 9.5 & 9.5 & Emulsion & 0.791 \\
2 & 9 & 9 & Emulsion & 0.762 \\
2.5 & 8.75 & 8.75 & Emulsion & 0.719 \\
3 & 8.5 & 8.5 & Emulsion & 0.677 \\
3.5 & 8.25 & 8.25 & Emulsion & 0.77 \\
4 & 8 & 8 & Emulsion & 0.296 \\
4.5 & 7.75 & 7.75 & Emulsion & 0.357 \\
5 & 7.5 & 7.5 & Emulsion & 0.884 \\
5.5 & 7.25 & 7.25 & Emulsion & 0.56 \\
6 & 7 & 7 & Emulsion & 0.969 \\
8 & 6 & 6 & Emulsion & 1.05 \\
10 & 5 & 5 & Emulsion & 1.265 \\
\hline
\end{tabular}

Table 3: Composition of different felodipine $(10 \mathrm{mg})$ liquisolid tablets prepared using PEG 400 as liquid medication for F1, SEDDS as liquid medication for F2 and F3 as a control tablet.

\begin{tabular}{llll}
\hline & \multicolumn{3}{c}{ Formulations } \\
\cline { 2 - 4 } & \multicolumn{1}{c}{ F1 } & \multicolumn{1}{c}{ F2 } & \multicolumn{1}{c}{ F3 } \\
\hline Liquid system & PEG 400 & SEDDS & ------ \\
Cd \% w/w & $10 \%$ & $10 \%$ & ----- \\
$\mathrm{R}$ & 10 & 10 & 10 \\
$\mathrm{~L}_{\mathrm{f}}$ & 0.331 & 0.196 & 0.331 \\
$\mathrm{~W}(\mathrm{gm})$ & 0.1 & 0.1 & ------ \\
Avicel (gm) & 0.302 & 0.51 & 0.302 \\
Aerosil 200(gm) & 0.0302 & 0.051 & 0.0302 \\
Croscarmellose(gm) & 0.0216 & 0.033 & 0.0216 \\
Total weight(gm) & 0.453 & 0.694 & 0.363 \\
Release efficiency $(\%)$ & $84.2 \pm 1.1$ & $92.5 \pm 0.2$ & $43.6 \pm 0.9$ \\
\hline
\end{tabular}

While Cd\% is the concentration of the drug in the liquid medication, $\mathbf{R}$ is the ratio represents the amounts of carrier : coating materials in the formula, $\mathbf{L}_{\mathbf{r}}$ represents the liquid load factor for each formula and $\mathbf{W}$ is the weight of liquid medications. The release efficiency values of the drug from the unprocessed powder and the SEDDS were 27.4 and $93.1 \%$, respectively.

\section{Drug release from SEDDS}

Figures 1 shows the release profiles of felodipine from unprocessed powder and from the SEDDS system in which the drug was dispersed at a concentration of $10 \% \mathrm{w} / \mathrm{w}$. The calculated dissolution efficiency values are presented in Table 3. The release of felodipine from the pure unprocessed powder was slow. Only $10.6 \%$ of the labeled amount was dissolved in the first 5 minutes followed by slow release over the time course of the study with a maximum of $37.5 \%$ of the being released after 2 hours. The overall dissolution efficiency of pure drug was only $27.4 \%$ (Figure 1 and Table 3). This release pattern is similar to the previously recorded release profile for the same drug (Karavas et al., 2006). The recorded slow release pattern is considered responsible for the variable and low bioavailability of felodipine after oral administration (Bhole and Patil., 2010). Incorporation of the drug in various SEDDS formulations resulted in significant increase in the dissolution rate. This is indicated from the amount of the drug released in the first 5 minutes which was $95.24 \%$. The overall dissolution efficiency was $93.14 \%$ (Figure 1). This rapid release pattern is advantageous for poorly soluble drugs especially those 
that are subject to first pass metabolism. This enhanced dissolution pattern can be explained on the bases that the SEDDS is dispersed in the dissolution medium forming microemulsion or coarse emulsion with small droplet size. This will result in significant increase in the surface area into which the drug can be dissolved. The high solubility of the drug in the selected components of SEDDS provides another explanation. This differentiates the system from the unprocessed drug which is in crystalline state that dissolves slowly in the dissolution medium.

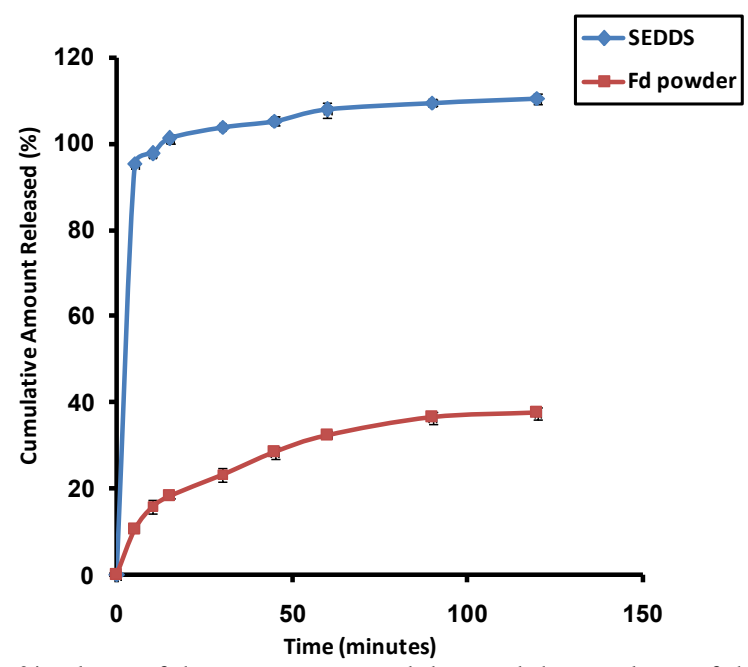

Fig 1: \% release of the pure unprocessed drug and the \% release of the drug from SEDDS.

\section{Formulation of liquisolid tablet}

The theory of liquisolid technique suggests that after incorporation of drug solution into a carrier material both absorption and adsorption processes take place. The process starts by initial absorption of the liquid by internal structure of the porous carrier. After the saturation of this process, adsorption of the liquid onto the internal and external surfaces of the porous carrier particles takes place. A coating material which has high adsorptive properties and large surface area is also included to produce the free flowing property of the liquisolid system (Fahmy and Kassem, 2008). To achieve this, Avicel PH 102 was thus selected as the carrier with Aerosil 200 being employed as the coating system.

\section{Determination of Flowable Liquid Retension Potential ( $\Phi$ - value)}

Both non self emulsifying and self emulsifying liquisolid tablets were prepared. For the non self emulsifying tablets, PEG 400 which was able to dissolve significant amounts of the drug was employed as the liquid phase. This solvent is widely used in preparation of liquisolid tablets using the same carrier and coating materials. The $\Phi$-values of this liquid in case of Aerosil 200 and Avicel pH 102 are well established in literature. The reported $\Phi$ values for Aerosil 200 and Avicel pH 102 with PEG 400 are 3.26 and 0.005 respectively (Pardhi et al., 2010; Sathali and Deepa, 2013; Jabbar and Hussien, 2013). These values were utilized in the current study. For self emulsifying tablet it was necessary to determine the $\Phi$-values for the SEDDS. Figure 2 shows the relation between the angle of slide and the corresponding $\Phi$-values for Aerosil 200 and Avicel pH 102 after mixing with the SEDDS formula respectively. This relationship was used to calculate the $\Phi$-value at $33^{\circ}$ which was calculated as 1.354 and 0.0615 for Aerosil 200 and Avicel pH 102 respectively. These values were used to calculate the $\mathrm{L}_{\mathrm{f}}$ which was used to calculate the amount of solid to be used in formulation of liquisolid tablet. The details of the components of prepared liquisolid tablets are presented in Table 3.
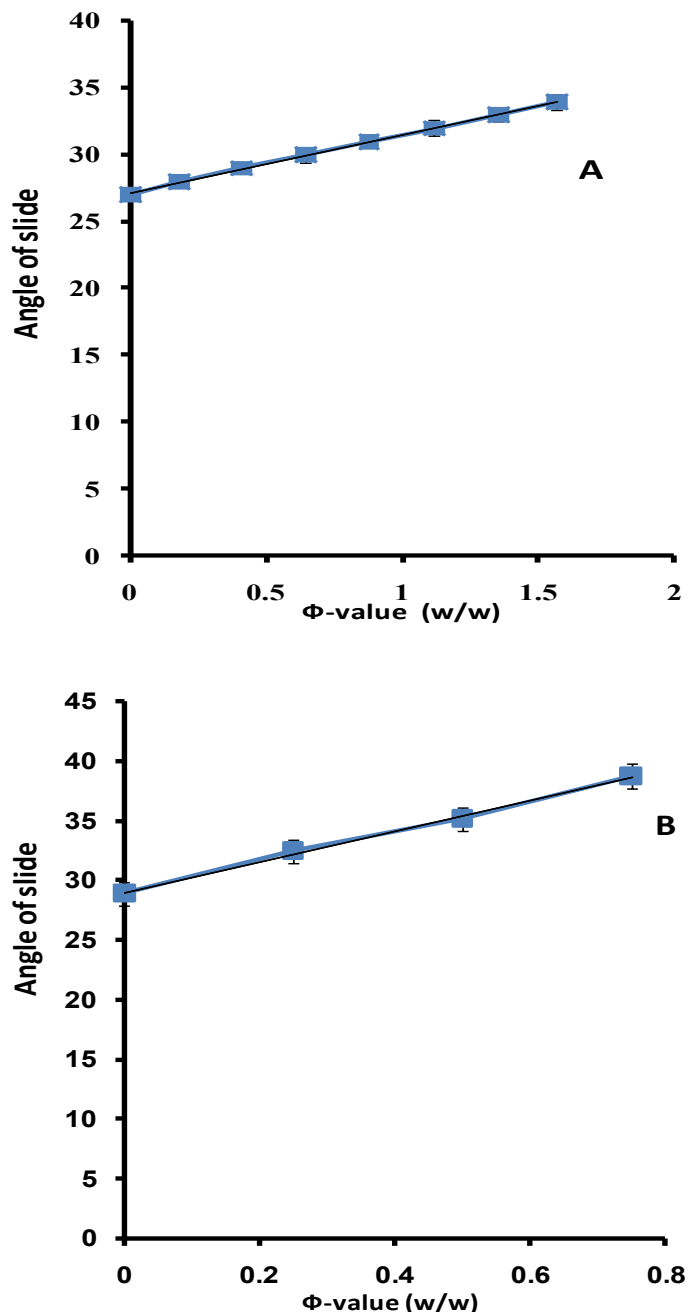

Fig. 2: The relationship between the angles of slide and $\Phi$-values for Aerosil 200 (A) and Avicel PH 102 (B) using SEDDS as the liquid .

\section{Evaluation of liquisolid tablets}

The prepared tablets were found to be of uniform weight with the recorded deviation from the average weight being in the range of $0.34-1.4 \%$. This reflects proper mixing and good flowability of the formulation. The recorded friability values were $0.133 \%$ for $\mathrm{F} 1,0.099 \%$ for $\mathrm{F} 2$ and $0.076 \%$ for $\mathrm{F} 3$. This is acceptable based on the acceptance criteria of the USP. The drug content was in the range $99.2 \pm 5.5 \%$ for $\mathrm{F} 1$ and $98.9 \pm 7.6 \%$ for 
$\mathrm{F} 2$ and $98.4 \pm 7.9 \%$ for $\mathrm{F} 3$. The disintegration time values were $45 \pm 1$ seconds indicating the efficiency of croscarmellose as superdisintegrant. The hardness of tablets was about $5 \pm 0.7 \mathrm{KP}$ for $\mathrm{F} 1, \mathrm{~F} 2$ and $\mathrm{F} 3$.

\section{Drug release from liquisolid tablet}

Figure 3 shows the release profile of felodipine from the prepared non-self emulsifying liquisolid tablet in which PEG 400 was used as the liquid medication and from self emulsifying liquisolid tablet in which SEDDS was the liquid vehicle compared to a standard tablet containing the same excipients except the liquid component. The release profiles were compared with that obtained from the unprocessed drug powder. The calculated release efficiency values are presented in Table 3. The unprocessed drug powder produced very slow release of the drug with only $16 \%$ of the drug being released in the first 10 minutes and a maximum of $37.5 \%$ being librated after 2 hours (Figure 3 ).

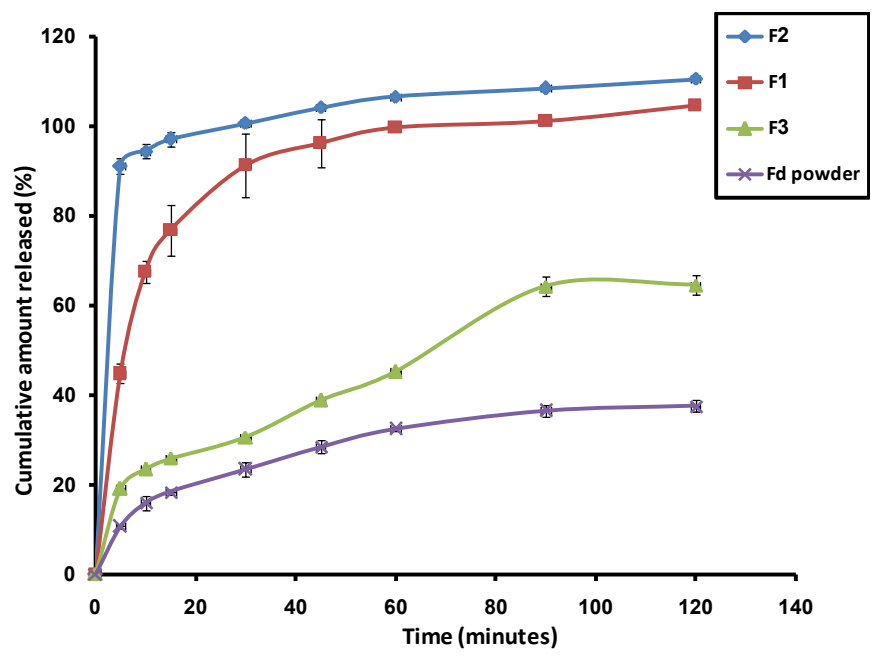

Fig. 3: The release profile of felodipine from the unprocessed drug ( $\mathrm{Fd}$ powder), non-self emulsifying tablet (F1), self emulsifying tablet (F2) and traditional (control) tablet (F3).

The calculated release efficiency of the drug from the unprocessed powder was $27.4 \%$. This erratic release is expected for such lipophilic drug and is well documented in literature as one of the most important factors reducing the oral bioavailability of felodipine (Bhole and Patil, 2009). Formulating this powder as standard tablet resulted in better release profile compared with the unprocessed drug powder but the release rate remained slower than required for optimum oral delivery. This tablet is considered as the control tablet in the current study. The control tablet librated only $23 \%$ of the dose in the first 10 minutes and librated $38.7 \%$ after 45 minutes with only $64.5 \%$ being released even after 2 hours. The recorded increase in the release of the drug from standard tablet compared to the unprocessed powder can be explained on the bases of possible adsorption of the drug on the large surface area of the carrier and coating materials (Avicel and Aerosil). This is expected to provide greater surface area from which better release was achieved. Nevertheless, the recorded release from this standard tablet is lower than required for a rapidly dissolving tablet. Formulating the drug as non-self emulsifying tablet resulted in a significant increase in the release rate compared with the standard tablet with the liquisolid tablet liberating $67 \%$ of the dose with the first 10 minutes (Figure 3). The calculated release efficiency from this liquisolid tablet was $84.2 \%$ (Table 3 ). The self emulsifying liquisolid tablets librated $94.3 \%$ of the dose within the first 10 minutes with the calculated release efficiency being $92.5 \%$ (Figure 3 and Table 3). These release parameters were significantly higher than that recorded in case of the standard tablets. It is important to note that the presence of superdisintegrant in the tablet formulation (F1, F2 and F3) was adequate resulting in fast disintegration (45 seconds) and this allowed rapid dissolution of the drug. Such short disintegration time eliminated the effect of compression. Similar behaviour was recorded for other lipophilic drugs which resulted in similar release pattern from the liquid formulation and the corresponding liquisolid system (Mahmoud et al., 2009; Kang et al., 2011). The recorded enhancement in the dissolution rate of the drug from the liquisolid formulations can be explained on the bases that the drug may exist in a solution form in the liquisolid system (to be confirmed by the thermal analysis studies, see below). This solution can mix with the dissolution medium with subsequent alteration in the saturation solubility of the drug in the diffusion layer surrounding the solid particles of the carrier into which the drug is adsorbed and/or absorbed. Accordingly, the enhanced dissolution from liquisolid systems can be explained on the bases of the "diffusion layer model" (Spireas et al., 1998) which derived from Noyes-Whitney equation which is described as follow:

$$
\mathrm{D}_{\mathrm{R}}=[\mathrm{DA}(\mathrm{Cs}-\mathrm{C})] / \mathrm{h}
$$

where, $\mathrm{D}_{\mathrm{R}}$ is the dissolution rate, $h$ is the thickness of the stagnant diffusion layer formed by the dissolving liquid around the drug particles, $D$ is the diffusion coefficient of the drug molecules transported through it, $A$ is the surface of drug available for dissolution, $C$ s is the saturation solubility of the drug in the diffusion layer, and $C$ is the drug concentration in the bulk of the dissolving medium (Spireas et al., 1998). Having conducted all the dissolution studies at a constant rotational paddle speed (50 rpm) and identical dissolution medium, it can be assumed that the thickness $(h)$ of the stagnant diffusion layer and the diffusion coefficient $(D)$ of the drug molecules remain almost identical. Accordingly, the observed higher dissolution rates of felodipine from liquisolid systems are due to the significantly increased surface of the molecularly dispersed felodipine (Javadzadeh et al., 2005). In addition, the saturation solubility of the drug in the microenvironment $(C s)$ might be increased in case of the liquisolid systems. The increase in CS will create higher concentration gradient and more driving force for drug dissolution. The later explains the efficacy of the liquisolid systems (Javadzadeh et al., 2005; Elkordy et al., 2013). The superiority of the self emulsifying tablet over the non-selfemulsifying tablet can be explained on the bases that the former disperses in the dissolution media forming emulsion which has greater surface area for drug release and diffusion. 


\section{Differential thermal analysis}

The liquisolid systems were subjected to differential thermal analysis (DTA). This was conducted with the goal of investigating the physical nature of the drug after formation of liquisolid system. To maximize the possibility of detecting the drug crystals if present in the liquisolid formulation, the drug solution was $(10 \% \mathrm{w} / \mathrm{v})$ was mixed with double its weight of solid. This produced dry product which was subjected to DTA. Figure 4 shows representative DTA traces of unprocessed drug, pure excipients and different liquisolid formulations. Pure felodipine showed a single, sharp endothermic peak at $146.45^{\circ} \mathrm{C}$ (Figure 4).
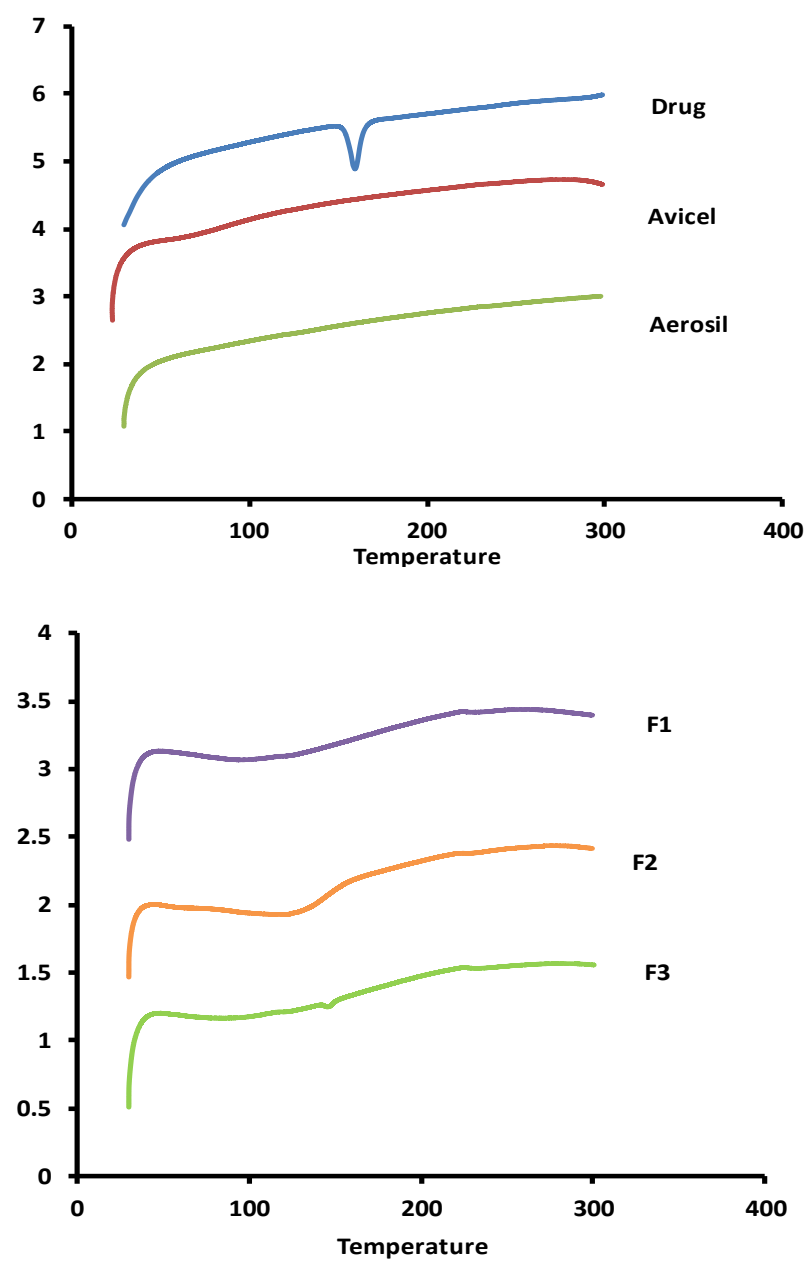

Fig. 4: Examples of the DTA traces of felodipine, Avicel, Aerosil 200 and various tablet mixtures in which the drug solutions in PEG 400 or in self emulsifying formulation $(10 \% \mathrm{w} / \mathrm{w})$ were mixed with double its weight of Avicel-Aerosil mixture (10:1) (F1 and F2, respectively). The control formulation involved mixing the drug $(10 \mathrm{mg})$ with $200 \mathrm{mg}$ of Avicel-Aerosil mixture (10:1) (F3).

This endothermic peak is attributed to the melting transition of the drug. Similar results were recorded by other investigator for the endothermic peak in a study performing the scanning up to $280{ }^{\circ} \mathrm{C}$ (Tapes et al., 2011). The thermograms of Avicel PH 102 displayed broad endothermic peak around $65{ }^{\circ} \mathrm{C}$ (Figure 4). This endotherm can be due to the volatilization of adsorbed water. The recorded thermogram complies with the published data by other investigators (Ibrahim et al., 2011). The thermal behavior of pure Aerosil 200 did not show any endothermic or exothermic peak in the range of temperature tested. This confirms that the coating material was almost in an amorphous state. Similar pattern was recorded for the same material (Sriamornsak et al., 2011; El-Gizawy et al., 2015).

The thermograms of the prepared tablets showed the broad endothermic peak corresponding to the absorbed moisture of avicel. The control formulation (F3) revealed small sharp endothermic at the same position of that obtained for the pure drug (Figure 4). This indicates that the drug was able to retain its crystalline nature after compression. The thermograms of the liquisolid tablets did not show any evidence for the endothermic peak of the drug. This indicates that the drug was in the form of solution in the liquisolid formulations. Similar conclusion was reported after thermal analysis of liquisolid tablet of other drug (Elkordy et al., 2013).

\section{CONCLUSION}

Liquisolid tablets formation can enhance the dissolution rate of felodipine. The nature of the liquid component is a factor affecting the release pattern of the drug from the liquisolid tablets. Self emulsifying liquid system was better than the simple liquid system for enhanced dissolution from liquisolid tablets.

\section{REFERENCES}

Ansari, KA, Pagar, KP, Anwar, S, Vavia, PR. Design and optimization of self-microemulsifying drug delivery system (SMEDDS) of felodipine for chronotherapeutic application. Braz J Pharm Sci, 2014; 50(1): 203-212

Basalious, EB, El-Sebaie, W, El-Gazayerly, O. Rapidly absorbed orodispersible tablet containing molecularly dispersed felodipine for management of hypertensive crisis: Development, optimization and in vitro/in vivo studies. Pharm Dev Tech, 2013; 18(2): 407-416

Bhole, PG, Patil, VR. Enhancement of water solubility if felodipine by preparing solid dispersion using polyethylene glycol 6000 and poly vinyl alchol. Asian J Pharm, 2009; 3(3): 240-244

Cardoza, RM, Amin, PD. A stability indicating LC method for felodipine. J Pharm Biomed Anal, 2002; 27(5): 711-718

Charman, WN, Charman, SA, Rogge, MC, Wilson, TD, Dutko, FJ, Pouton, CW. Self-emulsifying drug delivery systems: formulation and biopharmaceutic evaluation of an investigational lipophilic compound. Pharm Res, 1992; 9(1):87-89

El-Gizawy, SA, Osman, MA, Arafa, MF, El Maghraby, GM. Aerosil as a novel co-crystal co-former for improving the dissolution rate of hydrochlorothiazide. Int J Pharm, 2015; 478(2): 773-778

Elkordy, AA, Tan, XN, Essa, EA. Spironolactone release from liquisolid formulations prepared with Capryol ${ }^{\mathrm{TM}}$ 90, Solutol HS-15 and Kollicoat SR 30 D as non-volatile liquid vehicles. Eur J Pharm Biopharm, 2013; 83(2):203-223

El Maghraby, GM, Elsergany, RN. Fast disintegrating tablets of nisoldipine for intra-oral administration. Pharm Dev Technol, 2014; 19(6):641-650

Fahmy, RH, Kassem, MA. Enhancement of famotidine dissolution rate through liquisolid tablets formulation: In vitro and in vivo evaluation. Eur J Pharm Biopharm, 2008; 69(3):993-1003

Gangineni, R, Sontha, P, Konda, SK. Formulation, characterization and evaluation of self emulsifying drug delivery system of repaglinide. J Pharm Res, 2013; 1(7):665-672 
Hariprasanna, RC, Upendra, K, Qamar, JA, Gururaj, SK, Srinath, BA .Study on formulation and processing factor influencing the release of felodipine. Int J Curr Pharm Res, 2010; 2(3):86-89

Hauss, JD. Oral lipid-based formulations. Adv Drug Deliv Rev, 2007; 59(7):667-676

Ibrahim, EH, El-Faham, TH, Mohammed, FA, El-Eraky, NS. Enhancement of solubility and dissolution rate of domperidone by utilizing different techniques. Bull Pharm Sci, 2011; 34(2):105-120

Jabbar, ASA, Hussien, AA. Formulation and evaluation of piroxicam liquisoild compacts. Inter J Pharm Pharm Sci, 2013; 5(1):132141

Javadzadeh, Y, Musaalrezaei, L, Nokhodchi, A. Liquisolid technique as a new approach to sustain propranolol hydrochloride release from tablet matrices. Int J Pharm, 2008; 362:102-108

Javadzadeh, Y, Siahi-Shadbad, MR, Barzegar-Jalali, M, Nokhodchi, A. Enhancement of dissolution rate of piroxicam using liquisolid compacts. IL Pharmaco, 2005; 60(4):361-365

Kang, MJ, Jung, SY, Song, WH, Park, JS, Choi, SU, Oh, KT, Choi, HK, Choi, YW, Lee, J, Lee, BJ, Chi, SC. Immediate release of ibuprofen from Fujicalin ${ }^{\circledR}$-based fast-dissolving self-emulsifying tablets. Drug Dev Ind Pharm, 2011; 37(11):1298-1305

Karavas, E, Georgarakis, E, Bikiaris, D. Felodipine nanodispersions as active core for predictable pulsatile chronotherapeutics using PVP/HPMC blends as coating layer. Int J Pharm, 2006; 313(12):89-197

Khaled KA. Formulation and evaluation of hydrochlorothiazide liquisolid tablets. Saudi Pharm J, 1998; 6:39-46

Kim CK, Park JS. Solubility enhancers for oral drug delivery; can chemical structure manipulation be avoided. Am J Drug Del, 2004; 2(2):113-130

Kuchekar, BS, Narkhede, MR. The effect of water soluble polymers on felodipine aqueous solubility and complexing abilities with natural and modified $\beta$-cyclodextrin. Iran J Pharm Sci, 2007; 3(4):197-202

Mahmoud, EA, Bendas, ER, Mohamed, MI. Preparation and evaluation of self-nanoemulsifying tablets of carvedilol. AAPS PharmSciTech, 2009; 10(1):183-192

Nokhodchi, A, Aliakbar, R, Desai, S. Liquisolid compacts: The effect of cosolvent and HPMC on theophylline release. Colloids and Surfaces, 2010;79(1):262-269

Pardhi DM, Shivhare UD, Mathur VB, Bhusari KP. Liquisolid technique for enhancement of dissolution properties of carvedilol. Der Pharmacia Letter, 2010; 2(5):412-42
Saltiel, E, Ellrodt, AG, Monk, JP, Langley, MS. Felodipine. A review of its pharmacodynamic and pharmacokinetic properties, and therapeutic use in hypertension. Drugs, 1988; 36(4):387-428

Sathail, AH, Deepa, C. Formulation of liquisolid tablets of candesartan cilexetil. Res Pharm Sci, 2013; 4(2):238-249

Singh, AK, Chaurasiya, A, Mishra, G, Asati, D, Khar, RK, Mukherjee, R. Oral bioavailabiility enhancement of exemestane from selfmicroemulsifying drug delivery system (SMEDDS). AAPS Pharmscitech, 2009; 10(3): 906-916

Spireas, S and Bolton, S. Sustained-release liquisolid compacts, In: 25th International Symposium on Controlled Release of Bioactive Materials, Nevada, USA, pp, 1998; 138-139

Spireas, SS, Jarowski, CI, Rohera, BD. Powdered solution technology: principles and mechanism. Pharm Res, 1992; 9(10): 13511358

Spireas, SS, Sadu, S. Enhancement of prednisolone dissolution properties using liquisolid compacts. Int J Pharm, 1998; 166(2):177-188

Spireas, S, Sadu, S, Grover, R. In vitro release evaluation of hydrocortisone liquisolid tablets. AAPS Pharmscitech, 1998; 87:867-872

Spireas, SS, Wang, T, Grover, R. Effect of powder substrate on the dissolution properties of methylclothiazide liquisolid compacts. Drug Dev Ind Pharm, 1999; 25(2):163-168

Sriamornsak, P, Kontong, S, Weerapol, Y, Nunthanid, J, Sungthongjeen, S, Limmatvapirat, S. Manufacture of ternary solid dispersions composed of nifedipine, eudragit e and adsorbent. Adv Mat Res, 2011; 317-319:185-188

Tapes, AR, Kawtikwar, PS, Sakarkar, DM. Modification of felodipine properties using spherically agglomerated solid dispersion. Am J Drug Discov Dev, 2011; 1(3):160-173

Tayel, SA, Soliman, II, Louis, D. Improvement of dissolution properties of Carbamazepine through application of the liquisolid tablet technique. Eur J Pharm Biopharm, 2008; 69(1): 342-347

United States Pharmacopiae, National Formulary 32, 2009.

\section{How to cite this article:}

Khorshed GS, El-Maghraby GM. Self microemulsefying and nonself microemulsefying liquisolid tablet of felodipine. J App Pharm Sci, 2016; 6 (07): 125-132. 